\title{
AN Fe XXIV ABSORPTION LINE IN THE PERSISTENT SPECTRUM OF THE DIPPING LOW-MASS X-RAY BINARY 1A 1744-361
}

\author{
Fotis P. Gavrill ${ }^{1,3}$, Tod E. Strohmayer ${ }^{1}$, and Sudip Bhattacharyya ${ }^{2}$ \\ ${ }^{1}$ NASA Goddard Space Flight Center, Astrophysics Science Division, Code 662, Greenbelt, MD 20771, USA \\ ${ }^{2}$ Department of Astronomy and Astrophysics, Tata Institute of Fundamental Research, Mumbai 400005, India \\ Received 2009 September 9; accepted 2012 April 21; published 2012 June 7
}

\begin{abstract}
We report on Chandra X-ray Observatory (Chandra) High Energy Transmission Grating spectra of the dipping low-mass X-ray binary 1A 1744-361 during its 2008 July outburst. We find that its persistent emission is well modeled by a blackbody $(k T \sim 1.0 \mathrm{keV})$ plus power law $(\Gamma \sim 1.7)$ with an absorption edge. In the residuals of the combined spectrum, we find a significant absorption line at $6.961 \pm 0.002 \mathrm{keV}$, consistent with the Fe XXVI (hydrogen-like Fe) 2-1 transition. We place an upper limit on the velocity of a redshifted flow of $v<221 \mathrm{~km} \mathrm{~s}^{-1}$. We find an equivalent width for the line of $27_{-3}^{+2} \mathrm{eV}$, from which we determine a column density of $(7 \pm 1) \times 10^{17} \mathrm{~cm}^{-2}$ via a curve-of-growth analysis. Using XSTAR simulations, we place a lower limit on the ionization parameter of $>10^{3.6} \mathrm{erg} \mathrm{cm} \mathrm{s}^{-1}$. We discuss what implications the feature has on the system and its geometry. We also present Rossi X-ray Timing Explorer data accumulated during this latest outburst and, via an updated color-color diagram, clearly show that 1A 1744-361 is an "atoll" source.
\end{abstract}

Key words: binaries: general - line: identification - stars: individual (1A 1744-361) - stars: neutron - X-rays: binaries - X-rays: stars

Online-only material: color figures

\section{INTRODUCTION}

1A $1744-361$ is a neutron star low-mass X-ray binary (LMXB) discovered by the Ariel $V$ satellite (Davison et al. 1976; Carpenter et al. 1977). This source is a transient LMXB, and several outbursts have been observed with a number of missions, most notably the Rossi X-ray Timing Explorer (RXTE). In 2001, Emelyanov et al. (2001) discovered a burst from the direction of 1A 1744-361, however, it could not be unambiguously identified as a thermonuclear burst. The first thermonuclear (Type I) burst from 1A 1744-361 was discovered by Bhattacharyya et al. (2006a) using RXTE. This Type I X-ray burst exhibited a $530 \mathrm{~Hz}$ burst oscillation which provided a measurement of the neutron star's spin frequency. The burst also provided an upper limit of $d<9 \mathrm{kpc}$, under the assumption that its maximum luminosity could not exceed the Eddington luminosity for a $1.4 M_{\odot}$ neutron star (Bhattacharyya et al. 2006a).

This source also shows regular incidents of intensity "dips" in its X-ray emission. According to Frank et al. (1987), such dips are produced by obscuring material associated with a structured accretion disk. Dips would only be visible for inclination angles $60^{\circ} \lesssim i \lesssim 80^{\circ}$ (Frank et al. 1987). In principle, dips could occur every orbital cycle, however, because of variations in the obscuring material in the disk, the presence of dips often varies from cycle to cycle and from source to source. The dips observed in 1A 1744-361, as is the case for other dipping LMXBs, exhibit complex structure (see Bhattacharyya et al. 2006a, Figure 4). By measuring the spacing between two closely spaced dip episodes observed by RXTE, Bhattacharyya et al. (2006a) estimated the orbital period of $1 \mathrm{~A} 1744-361$ to be $97 \pm 22$ minutes.

Narrow absorption features have been seen in the persistent emission of many "dipping" LMXBs. For example, 4U 1916-05 (Balman 2009; Iaria et al. 2006; Juett \&

\footnotetext{
3 Also at CRESST, University of Maryland Baltimore County, Baltimore, MD 21250, USA.
}

Chakrabarty 2006; Boirin et al. 2004), XB 1624-490 (Xiang et al. 2009; Balman 2009; Iaria et al. 2007), 4U 1323-62 (Bałucińska-Church et al. 2009; Balman 2009; Church et al. 2005; Boirin et al. 2005), 4U 1746-371 (Balman 2009), and EXO 0748-676 (Jiminez-Garate et al. 2003; Church et al. 1998). By far, the most prominent features are the Fe XXV (He-like) and Fe xxvi (H-like) lines. These features allow one to probe the structure, dynamics, and evolution of the material surrounding the neutron star. Thus, the spectral properties of 1A 1744-361 are of great interest. Using RXTE data, Bhattacharyya et al. (2006b) found that the persistent spectrum of this source is well modeled by a Comptonized blackbody model (Bhattacharyya et al. 2006b), and they also found evidence of a broad $(\sim 0.6 \mathrm{keV})$ iron emission feature at $\sim 6 \mathrm{keV}$ and an iron absorption edge at $\sim 8 \mathrm{keV}$. RXTE is not sensitive to narrow spectral features given its coarse spectral resolution and large FOV, thus, during the 2008 July outburst of this source, we triggered Chandra X-ray Observatory (Chandra) target-ofopportunity (ToO) observations for this purpose.

In Section 2, we present our analysis of the Chandra (Section 2.1) and RXTE (Section 2.2) data from 1A 1744-361. In Section 2.1.1, we present high-resolution spectra of the source in which we find an Fe XXVI absorption feature. In Section 2.2.1, we present an updated color-color diagram, and in Section 3 we discuss our results.

\section{ANALYSIS}

\subsection{Chandra Observations}

During 1A 1744-361's last outburst in 2008 July, we triggered three Chandra ToO Observations. These observations are summarized in Table 1. In order to search for narrow absorption features such as those seen in other dipping LMXBs, the data were taken with the High Energy Transmission Grating (HETG; Canizares et al. 2005) aboard Chandra. The HETG consists of two arms, the High Energy Grating (HEG) and the Medium Energy Grating (MEG). The HEG is sensitive to 
Table 1

1A 1744-361 Observation Log and Spectral Properties

\begin{tabular}{|c|c|c|c|c|}
\hline Parameter $^{\mathrm{a}}$ & 2008 Jul 5 & 2008 Jul 6 & $2008 \mathrm{Jul} 7$ & Total \\
\hline Obs. ID & 9884 & 9885 & 9042 & $\ldots$ \\
\hline Exposure (ks) & 24.2 & 21.0 & 30.4 & 75.6 \\
\hline Count rate (counts s ${ }^{-1}$ ) & $4.9 \pm 0.02$ & $4.86 \pm 0.02$ & $5.02 \pm 0.02$ & $4.927 \pm 0.009$ \\
\hline \multicolumn{5}{|c|}{ Continuum model } \\
\hline$N_{\mathrm{H}}^{\mathrm{b}}\left(\times 10^{22} \mathrm{~cm}^{-2}\right)$ & $0.40_{-0.03}^{+0.03}$ & $0.35_{-0.03}^{+0.03}$ & $0.44_{-0.03}^{+0.03}$ & $0.414_{-0.015}^{+0.006}$ \\
\hline$E_{\text {Edge }}{ }^{c}(\mathrm{keV})$ & $7.56_{-0.05}^{+0.08}$ & $7.73_{-0.01}^{+0.01}$ & $7.699_{-0.008}^{+0.013}$ & $7.645_{-0.015}^{+0.006}$ \\
\hline$\tau_{\text {Edge }}{ }^{d}$ & $1.8_{-0.2}^{+0.3}$ & $3.5_{-0.7}^{+1.3}$ & $3.5_{-0.6}^{+1.3}$ & $1.17_{-0.09}^{+0.10}$ \\
\hline$k T_{\mathrm{bb}}{ }^{\mathrm{e}}(\mathrm{keV})$ & $0.98_{-0.08}^{+0.08}$ & $1.05_{-0.08}^{+0.11}$ & $1.09+0.06$ & $1.06_{-0.03}^{+0.02}$ \\
\hline$R_{\mathrm{bb}}{ }^{\mathrm{f}}(\mathrm{km})$ & $4.8_{-0.7}^{+0.8}$ & $4.4_{-0.6}^{+0.7}$ & $4.4_{-0.3}^{+0.4}$ & $4.3_{-0.2}^{+0.2}$ \\
\hline$L_{\text {bol }}{ }^{\mathrm{g}}\left(\times 10^{36} \mathrm{erg} \mathrm{s}^{-1}\right)$ & $2.6_{-0.3}^{+0.3}$ & $3.0_{-0.4}^{+0.6}$ & $3.5_{-0.4}^{+0.5}$ & $3.1_{-0.2}^{+0.2}$ \\
\hline$\Gamma_{\mathrm{PL}}^{\mathrm{h}}$ & $1.64_{-0.06}^{+0.06}$ & $1.51_{-0.05}^{+0.07}$ & $1.80_{-0.06}^{+0.07}$ & $1.68_{-0.02}^{+0.02}$ \\
\hline Power-law flux ${ }^{\mathrm{i}}\left(\times 10^{-9} \mathrm{erg} \mathrm{cm}^{-2} \mathrm{~s}^{-1}\right)$ & $1.26_{-0.04}^{+0.04}$ & $1.39_{-0.06}^{+0.04}$ & $1.14_{-0.06}^{+0.05}$ & $1.25_{-0.02}^{+0.03}$ \\
\hline Total flux ${ }^{\mathrm{j}}\left(\times 10^{-9} \mathrm{erg} \mathrm{cm}^{-2} \mathrm{~s}^{-1}\right)$ & 1.399 & 1.368 & 1.241 & 1.367 \\
\hline$\chi_{\mathrm{DoF}}^{2}[\mathrm{DoF}]$ & $0.70[1490]$ & $0.69[1333]$ & $0.77[1883]$ & $0.83[3407]$ \\
\hline \multicolumn{5}{|c|}{ Fe XXVI line properties } \\
\hline$\overline{E_{i}^{\mathrm{k}}}(\mathrm{keV})$ & $6.97 \pm 0.03$ & $\ldots$ & $6.97 \pm 0.23$ & $6.961 \pm 0.002$ \\
\hline$\Delta E^{\mathrm{l}}(\mathrm{eV})$ & $<50$ & $\cdots$ & $<229$ & $15.5 \pm 2.4$ \\
\hline$W_{E}^{\mathrm{m}}(\mathrm{eV})$ & $33_{-15}^{+18}$ & $\cdots$ & $<40$ & $27_{-3}^{+2}$ \\
\hline $\mathrm{FWHM}^{\mathrm{n}}\left(\mathrm{km} \mathrm{s}^{-1}\right)$ & $<23192$ & $\cdots$ & $<5065$ & $1613 \pm 21$ \\
\hline$N^{\mathrm{o}}\left(\times 10^{17} \mathrm{~cm}^{-2}\right)$ & $10_{-6}^{+12}$ & $\cdots$ & $<13$ & $7.4_{-1.0}^{+1.1}$ \\
\hline
\end{tabular}

Notes.

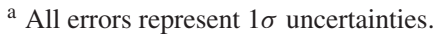

b Hydrogen column density.

c Absorption edge energy threshold.

${ }^{\mathrm{d}}$ Maximum depth of absorption edge at energy threshold.

e Blackbody temperature.

${ }^{\mathrm{f}}$ Blackbody radius as determined from the normalization of the blackbody component and assuming a distance of $d=9$ kpc (the upper limit on the distance to the source).

$\mathrm{g}$ Bolometric luminosity as determined from the normalization of the blackbody component and assuming a distance of $d=9 \mathrm{kpc}$ (the upper limit on the distance to the source).

$\mathrm{h}$ Index of power-law component.

${ }^{\mathrm{i}}$ Unabsorbed 2-10 keV flux of the power-law component.

$\mathrm{j}$ Unabsorbed 2-10 keV flux of the combined model.

${ }^{\mathrm{k}}$ Line energy, see Equation (1).

${ }^{1}$ Line width, see Equation (1).

${ }^{m}$ Equivalent width of the line.

${ }^{n}$ FWHM of the line in units of velocity.

${ }^{\circ}$ Column density of the ion as determined by a curve-of-growth analysis, see Section 2.1.2 for details.

photons in the $0.8-10 \mathrm{keV}$ band with a resolving power of $E / \Delta E \sim 1000$ at $1 \mathrm{keV}$, and the MEG is sensitive to photons in the $0.4-5.0 \mathrm{keV}$ band with a resolving power of 660 at $0.8 \mathrm{keV}$. The gratings were operated with ACIS, which enables separation of the individual diffraction orders, $m= \pm 1, \pm 2, \pm 3$, and a non-dispersed zeroth-order image is also obtained. We inspected all dispersed orders; however, for our analysis, we concentrated on the $|m|=1$ data because its effective area is more than an order of magnitude greater than the other orders for both the MEG and the HEG. Data in the different orders can be combined, however, they have different spectral and spatial responses and thus we avoided doing so, lest we smear out any narrow features.

\subsubsection{High-resolution Spectroscopy}

For each HETG arm and dispersed order, we extracted a source and background spectrum starting from the level 2 event file. We then created a response matrix and ancillary response file for each arm and order following the standard Chandra analysis threads. We then grouped the spectra so that there were no less than 60 counts per bin after background subtraction. We used XSPEC ${ }^{4}$ v12.5.1 for subsequent spectral modeling. We fit the MEG data to a photoelectrically absorbed blackbody and power-law model. The MEG data was well fitted by this model, and there were no significant deviations. We then analyzed the HEG data and fitted the same spectral model with the addition of an absorption edge at $\sim 7.6 \mathrm{keV}$, because of the HEG's higher response to photons above $5 \mathrm{keV}$. The addition of the absorption edge was not physically motivated, rather it was included because it provided a better empirical model of the continuum. For the HEG, we ignored bins above $8 \mathrm{keV}$ because of their diminished statistics. A photoelectrically absorbed blackbody+powerlaw+edge model fits well, see Table 1, however, the residuals showed a clear deviation from the model at $6.96 \mathrm{keV}$ in the 2008 July 5 (Figure 1, panels (1A) and (1B)) and 2008 July 7

\footnotetext{
4 http://xspec.gsfc.nasa.gov
} 


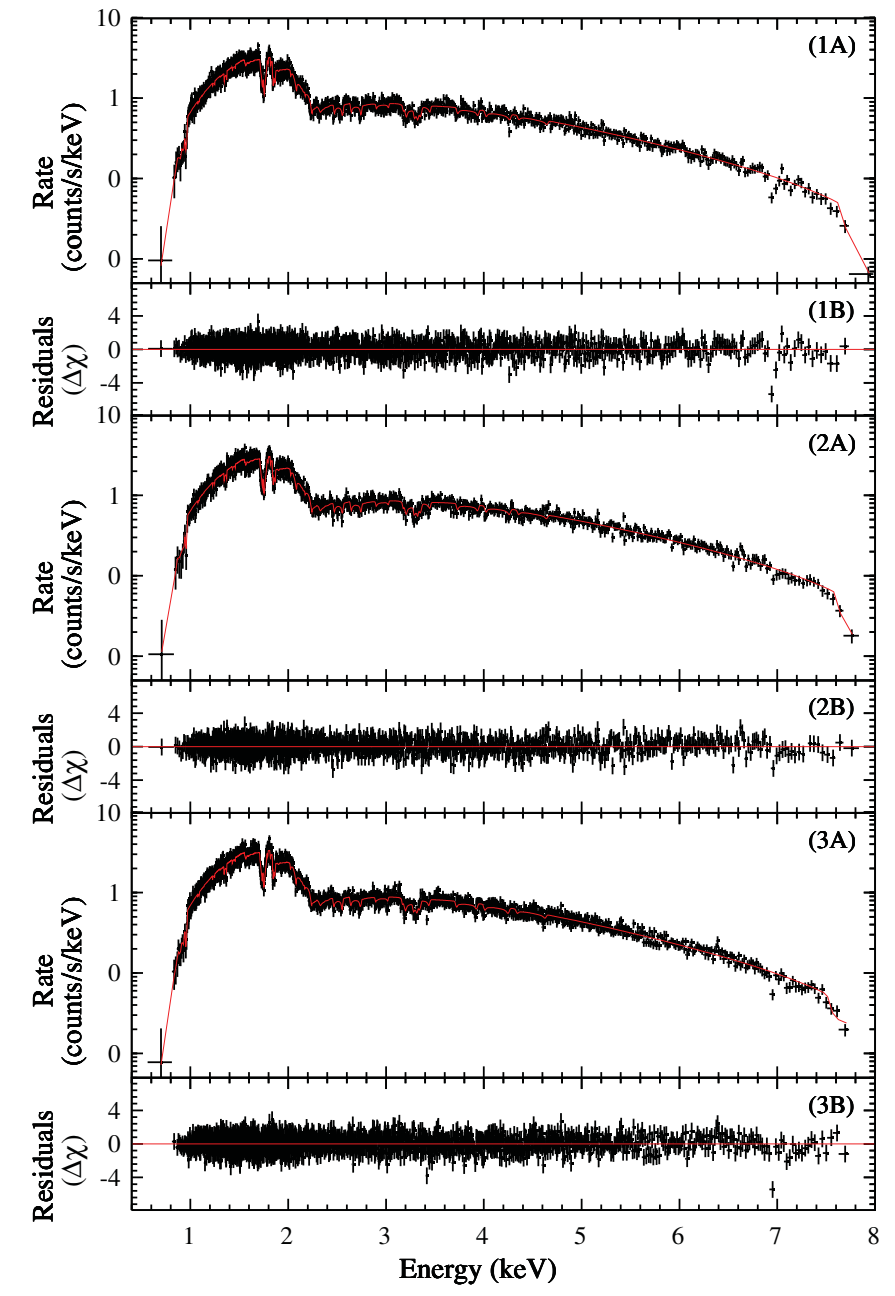

Figure 1. HEG order $m=-1$ spectra of 1A 1744-361. Panels (1A), (2A), and (3A) correspond to the 2008 July 5 , July 6 , and July 7 observations, respectively. The red curve represents the best-fit spectral model, see Table 1 for details. Panels (1B), (2B), and (3B) display the residuals, in terms of $\sigma$ from the model, after fitting for the best-fit spectral model. Notice the highly significant deviations from the model at $6.961 \mathrm{keV}$ in panels (1B) and (3B).

(A color version of this figure is available in the online journal.)

(Figure 1, panels (2A) and (2B)) observations. There were no comparable deviations in the 2008 July 6 observation, however, a hint of the feature could be seen at $6.96 \mathrm{keV}$. We noticed that the feature was primarily in the $m=-1$ and not the $m=1$ spectra, in principle the two should have comparable spectra, since they have comparable responses and effective areas in that energy range, however, dithering could affect the spectrum and smear out the feature, which is what we suspect happened to the order of $m=1$ spectrum. We do not believe that the feature is an instrumental artifact because then it would be seen in all $m=-1$ HETG observations of 1A 1744-361.

In order to increase the signal-to-noise ratio of the feature, we combined all three observations and their ancillary response files. The combined spectrum is displayed in Figure 2, and the $6.96 \mathrm{keV}$ feature can be clearly seen (Figure 2, inset). In the combined spectrum, the line is clearly resolved. Assuming that the line is broadened because of the thermal Doppler effects, then the line profile close to the peak should resemble a Gaussian:

$$
\phi_{G}(E, k T)=\frac{1}{\sqrt{2 \pi} \Delta E} \exp \left[-\frac{\left(E-E_{i}\right)^{2}}{2 \Delta E^{2}}\right],
$$

Table 2

Theoretical Properties ${ }^{\mathrm{a}}$ of the Fe xxv (He-like) and Fe xxvi (H-like) Lines

\begin{tabular}{lccc}
\hline \hline Ion & $\begin{array}{c}E_{0}{ }^{\mathrm{b}} \\
(\mathrm{keV})\end{array}$ & $f^{\mathrm{c}}$ & $A_{u l} \mathrm{~d}^{\mathrm{d}}\left(\times 10^{14} \mathrm{~s}^{-1}\right)$ \\
\hline Fe XXV & 6.70010 & 0.775 & 5.033 \\
Fe XXVI & 6.96614 & 0.408 & 2.863 \\
\hline
\end{tabular}

Notes.

a All parameters were provided by the XSTAR online line finding list http://heasarc.gsfc.nasa.gov/docs/software/xstar/xstar.html.

${ }^{\mathrm{b}}$ Energy of transition.

c Oscillator strength.

${ }^{\mathrm{d}}$ Einstein coefficient.

where $E_{i}$ is the line energy of the ion, and the line width, $\Delta E$, is given by

$$
\Delta E=E_{i}\left(k T / m_{i} c^{2}\right)^{1 / 2},
$$

where $m_{i}$ is the ion mass and $k T$ is the kinetic temperature (see Rybicki \& Lightman 1979). Fitting Equation (1) times a normalization to the line profile, we obtain a line energy of $E_{i}=6.961 \pm 0.002 \mathrm{keV}$ and a standard deviation of $\Delta E=15.5 \pm 2.3 \mathrm{eV}$. The theoretical properties of the Fe XxV (He-like) and the Fe XXVI (H-like) lines, the most prevalent lines in dipping LMXB spectra, are listed in Table 2. These values were obtained from the online line finding list ${ }^{5}$ provided by the XSTAR emission modeling package (Kallman \& Bautista 2001). The peak energy is consistent with the Fe XXVI $n=2-1$ transition, and according to Equation (2), our measured line width corresponds to a kinetic temperature of $k T=254 \pm 2 \mathrm{keV}$. Using the difference between the observed, $E_{i}$, and rest energy, $E_{0}$, of the line (see Table 2), we can determine the velocity of the absorbing material via $\left(E_{i}-E_{0}\right) / E_{i}=v / c$, for which we find $v<221 \mathrm{~km} \mathrm{~s}^{-1}$. We can only place an upper limit of the flow velocity because the wavelength difference we measure $(\Delta \lambda=0.001 \AA)$ is less than the absolute wavelength accuracy $(\Delta \lambda=0.006 \AA)$ of the HEG, see the Chandra X-ray Center's Proposer's Observatory Guide. ${ }^{6}$ Given that our particular choice of grouping could affect the observed central energy of the observed Fe XXVI line, and thereby bias our flow velocity measurements, we verified that there were no discrepancies in the observed energy of the Fe Xxvi line between the grouped and ungrouped spectra. Fits to the ungrouped spectra for the two observations in which the line was observed, and for the combined spectrum, returned line energies that were consistent with those found from the grouped spectrum. Furthermore, the corresponding wavelength shift of the line in the ungrouped spectra was all below the absolute wavelength accuracy of the detector.

\subsubsection{Curve-of-growth Analysis}

A curve-of-growth (COG) analysis allows one to relate the equivalent width, $W_{E}$, of a spectral line to the column density, $N$, of the absorbing ion. XSPEC provides a function which computes the equivalent width of a line, for which we obtain $W_{E}=27_{-3}^{+2} \mathrm{eV}$. To verify our XSPEC-measured equivalent width, we also computed the equivalent width analytically, by assuming that in the spectral region of interest the continuum is dominated by the power-law component. Our analytically determined equivalent width, $29.8 \mathrm{eV}$, was consistent with the value found using XSPEC.

\footnotetext{
5 http://heasarc.gsfc.nasa.gov/docs/software/xstar/xstar.html

6 http://cxc.harvard.edu/proposer/POG/pdf/MPOG.pdf
} 


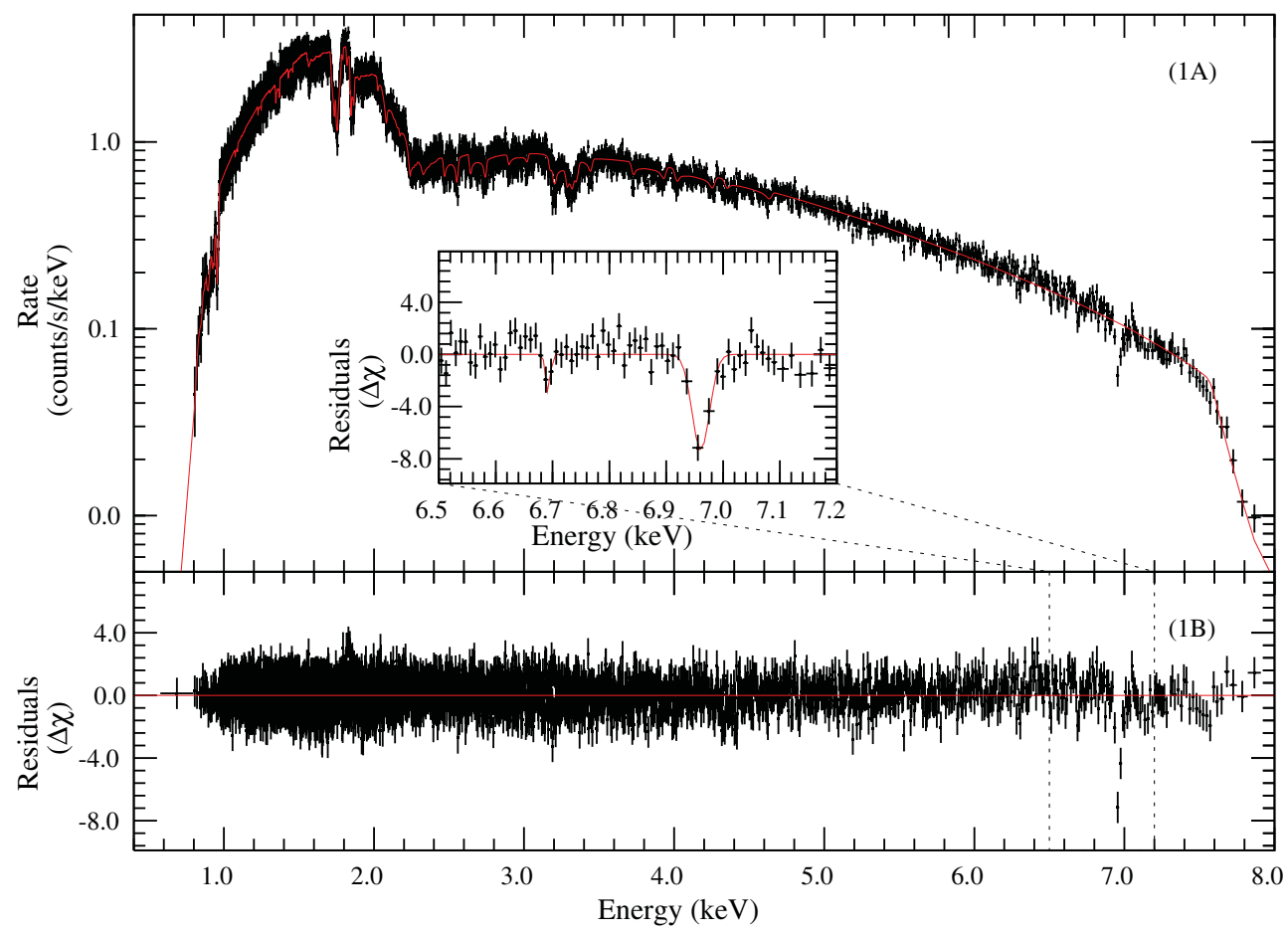

Figure 2. Sum of the three HEG $m=-1$ spectra of 1A 1744-361 displayed in Figure 1. The red curve is the best-fit model (photoelectrically absorbed blackbody plus power law times an edge, see the text for details). Panel (1B): the residuals, in terms of $\sigma$ from the model, after subtracting the best-fit model displayed in panel (1B). Notice the highly significant deviations from the model at $6.961 \mathrm{keV}$ corresponding to the Fe XxvI transition. Inset: an expanded view of the residuals around the Fe XXvI feature. The red curve is the best-fit Gaussian line profile, which has a centroid at $E_{0}=6.961 \pm 0.002 \mathrm{keV}$ with a width of $\Delta E=15.5 \pm 2.3 \mathrm{eV}$, see the text for details. The Fe xxv is not statistically significant, however, we fit for it in order to place an upper limit on its column density.

(A color version of this figure is available in the online journal.)

In order to calculate the column density, we computed theoretical COGs following Kotani et al. (2000) and Rybicki \& Lightman (1979). The equivalent width, $W_{E}$, of the line is related to the optical depth, $\tau$ via

$$
W_{E}=\int_{0}^{\infty}\left(1-e^{-\tau}\right) d E
$$

here $\tau=N s \phi$, where $N$ is the column density, $\phi$ is the line profile shape, and

$$
s=\pi f e^{2} / m_{i} c,
$$

where $f$ is the oscillator strength (see Table 2), and $m_{e}$ and $e$ are the electron mass and charge, respectively. Note that there is also a temperature-dependent term in Equation (4) which we ignored because Kotani et al. (2000) showed that the contribution of this term is negligible. The overall line profile, $\phi(E, k T)$ is found by convolving the line profile due to thermal broadening $\left(\phi_{G}\right.$, Equation (1)) and the line profile due to collisional broadening $\left(\phi_{L}\right)$, which has a Lorentzian profile, i.e.,

$$
\phi_{L}(E)=\frac{h \gamma}{4 \pi^{2}\left(E-E_{0}\right)^{2}+(h \gamma / 2)^{2}},
$$

where $\gamma$ is the sum of the Einstein coefficients, $A_{u l}$, over all lower energy states (see Table 2). Using the kinematic temperature found above, we then numerically integrated Equation (3) to obtain our COG, which is displayed in Figure 3. Using the error on the dynamic temperature and the equivalent width, we can obtain a confidence region for the Fe XxvI column density. Note that our feature appears to lie beyond the "linear regime" of the COG. We find $N_{\mathrm{Fe} \text { XXVI }}=7.4_{-1.0}^{+1.1} \times 10^{17} \mathrm{~cm}^{-2}$ for Fe XXVI.

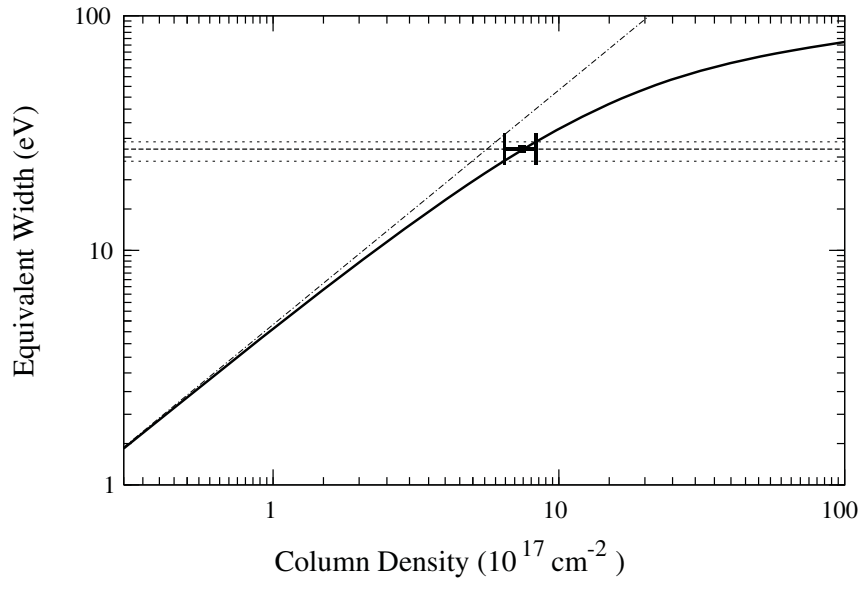

Figure 3. COG for the FexxvI absorption line observed in the persistent emission of 1A 1744-361: equivalent width vs. column density for a kinematic temperature of $k T=254 \mathrm{keV}$. The error on the COG due to the error on the temperature would be smaller than the thickness of the line. The horizontal dashed line indicates the position of our measured equivalent width, and the dotted lines are the upper and lower bounds on the equivalent width. The dash-dotted line represents the extrapolation of the "linear regime" of the COG.

\subsubsection{Ionization Parameter}

Although the presence of the Fe Xxvi feature is clear, there was no discernible Fe Xxv feature. However, an upper limit on the column density of the Fe XXv can constrain the ionization parameter $\xi$.

In order to place an upper limit on the equivalent width of the Fe XXV transition, we fitted for such a line using our combined spectrum, see Figure 2 (inset). Then, using XSPEC, we calculated 


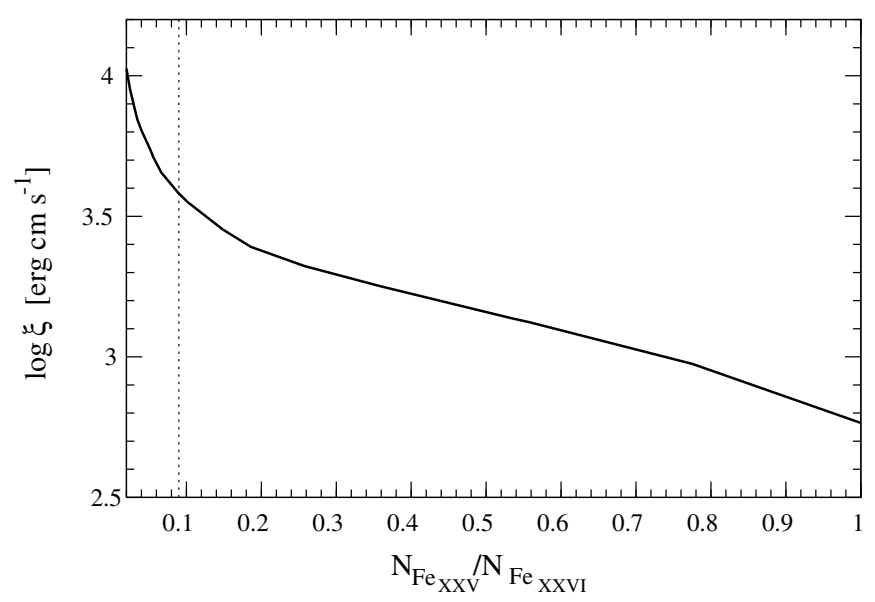

Figure 4. Ionization parameter, $\xi$, as a function of the relative abundance of Fe XXV to Fe XXVI as determined by our XSTAR simulations, see Section 2.1.3 for details. The vertical dotted lines represent our upper limit on the relative abundance of Fe XXV to Fe XXVI which places a lower limit on the ionization parameter of $\xi>10^{3.6} \mathrm{erg} \mathrm{cm} \mathrm{s}^{-1}$.

the equivalent width in the same manner as for Fe XXVI. We then generated a COG for Fe XXV using the corresponding oscillator strength and Einstein coefficient (see Table 2). From our COG analysis, we place an upper limit on the column density of Fe XXV of $N_{\mathrm{Fexxv}}<6.3 \times 10^{16} \mathrm{~cm}^{-2}$. To calculate $\xi$, we ran simulations of an ionized absorber using XSTAR. We assumed a spherical distribution of material and we used the standard spectral shape of a power law with a spectral index of two. We created a grid of ionization parameters and relative abundances using different initial choices for the electron density and luminosity. As an upper limit on the choice of luminosity, we used the bolometric luminosity as measured from the blackbody component, $L<3.1 \times 10^{36} \mathrm{erg} \mathrm{s}^{-1}$ (see Table 1). Our plot of $\xi$ as a function of the ratio of the column density of Fe XXV to Fe XXVI is shown in Figure 4. Given that the relative abundance of Fe XXV to Fe XXVI is less than 0.09, this places a lower limit on the ionization parameter of $\xi>10^{3.6} \mathrm{erg} \mathrm{cm} \mathrm{s}^{-1}$.

\subsection{RXTE Observations}

The high time resolution data presented here were obtained from the Proportional Counter Array (PCA) aboard RXTE. The PCA is made up of five independent proportional counter units (PCUs). Each PCU is made up of three xenon/methane layers and an uppermost propane veto layer. The PCUs are sensitive to photons in the $\sim 2-60 \mathrm{keV}$ band binned into 256 channels. The PCA can time tag a photon to an accuracy of $\sim 1 \mu \mathrm{s}$. RXTE provides two standard data modes. Standard -1 mode data provide light curves with $0.125 \mathrm{~s}$ temporal bins and are summed over all spectral channels. Standard-2 data provide light curves with $16 \mathrm{~s}$ time bins and are grouped into 129 spectral channels. As well as the standard modes, there are several user-selected modes available. The ones used here include GoodXenon and Event modes. GoodXenon mode provides the full temporal and spectral resolution. Event mode data provide high time resolution but often at slightly lower time resolution than GoodXenon, and the spectral channels are grouped using different grouping schemes, but often preserving 64 spectral channels.

\subsubsection{Color-Color Diagram}

We created a color-color diagram using the Standard-2 data. Each PCU has different energy response, and PCUs 0 and
1 have lost their veto propane layer. PCU 2 is the most reliable, thus, in our analysis we used only photons from the top Xenon layer of PCU 2. For count rates below 20 counts $\mathrm{s}^{-1}$, we used 2048 s long intervals, for count rates between 20 and 40 we took $512 \mathrm{~s}$ long intervals, and for the highest count rates we used $256 \mathrm{~s}$ long time intervals. For each spectrum, we generated a model background using the FTOOLpcabackest. We defined our colors as follows: our soft color is the ratio of counts in the $2.30-3.54 \mathrm{keV}$ band to that in the $3.54-5.19 \mathrm{keV}$ band, and our hard color is defined as the ratio of the counts in the 5.19-8.52 keV band to that in the 8.52-17.74 keV band. Our color-color diagram is displayed in Figure 5. In Bhattacharyya et al. (2006b), the authors reported that the source exhibited atoll-like behavior, however, a Z-source track could not be unambiguously ruled out. The additional RXTE data presented here clearly display atoll source behavior.

\subsubsection{Timing Analysis}

Bhattacharyya et al. (2006a) reported a tentative $(2.3 \sigma)$ detection of an $800 \mathrm{~Hz}$ quasi-periodic oscillation (QPO) for 1A 1744-361. Using all the available RXTE event data of 1A 1744-361, we searched for low-frequency and kHz QPOs. In our search, we broke up our light curves into $250 \mathrm{~s}$ intervals. We found no highly significant $(\gg 3 \sigma)$ QPOs. The most significant QPO we found was a low-frequency QPO at $29 \mathrm{~Hz}$ QPO for an observation on 2008 July 17 (Obs. ID 93155-01-06-03) at a significance of $3.0 \sigma$.

In the RXTE archive, we find a total of three thermonuclear bursts from 1A 1744-361. These bursts are displayed in Figure 6. The first burst occurred on 2005 July 16 (Obs. ID 91050-05-01-00) and was first reported by Bhattacharyya et al. (2006a). This burst is the brightest observed from this source thus far (see Figure 6, panel (a)). Using its luminosity, Bhattacharyya et al. (2006a) placed an upper limit on the distance to the source of $d<9 \mathrm{kpc}$. The second and third bursts occurred on 2008 June 24 and 2008 July 25, respectively (see Figure 6, panels (b) and (c)). Bhattacharyya et al. (2006a) had found a significant burst oscillation in the first burst. We searched the other bursts for burst oscillations but found no comparable signals.

\section{DISCUSSION}

In this paper, we presented Chandra HETG observations of the dipping LMXB 1A 1744-361 during its latest outburst. Although the source's persistent spectrum was well modeled by a simple continuum model, there was significant evidence for a narrow absorption feature at $6.96 \mathrm{keV}$. The feature is consistent with the wavelength of the Fe XXVI (hydrogen-like Fe) $n=2-1$ transition to the wavelength accuracy of the detector. We find no evidence for statistically significant lines due to other transitions. Here, we discuss the properties of the continuum and line emission and we discuss what implications both have on the geometry of the source. We also discuss the limitations of our data, compare this source to other X-ray dippers, and speculate on what we may learn from future observations.

\subsection{The Continuum Spectrum of 1A 1744-361}

The continuum spectra of dipping LMXBs are generally well modeled by two components, one due to emission near the surface of the neutron star, and a Comptonized component possibly due to an extended Accretion Disk Corona (ADC: see Church \& Bałucińska-Church 2004). Often, a blackbody 


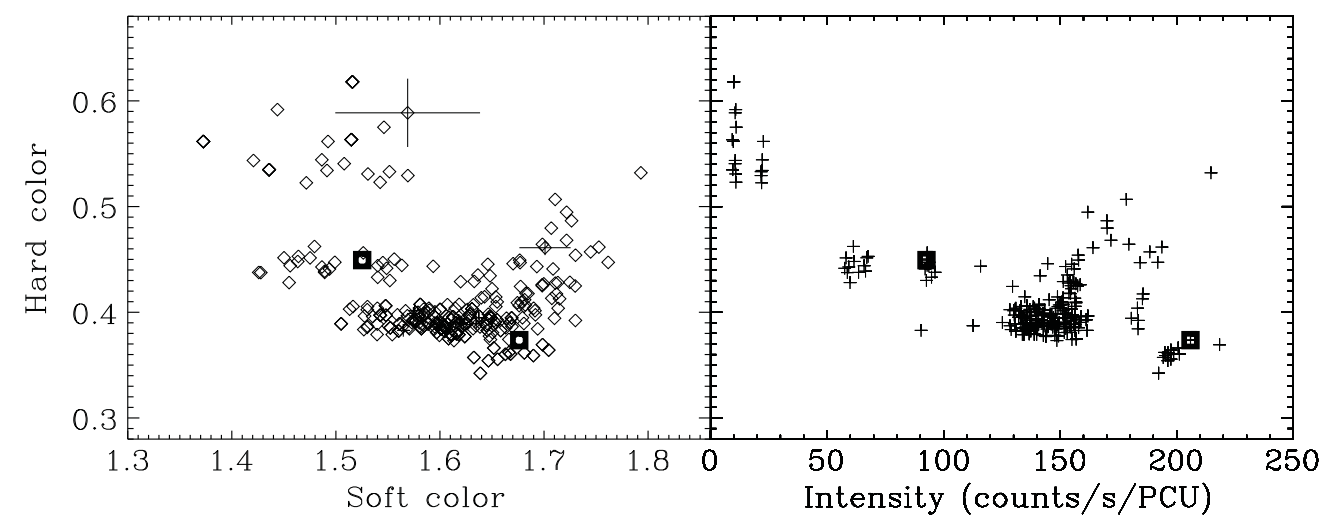

Figure 5. Color-color diagram of 1A 1744-361 as observed by RXTE. Left: hard color vs. soft color, see Section 2.2 .1 for details. Right: hard color vs. intensity. From both panels notice that 1A 1744-361 exhibits atoll-like behavior.

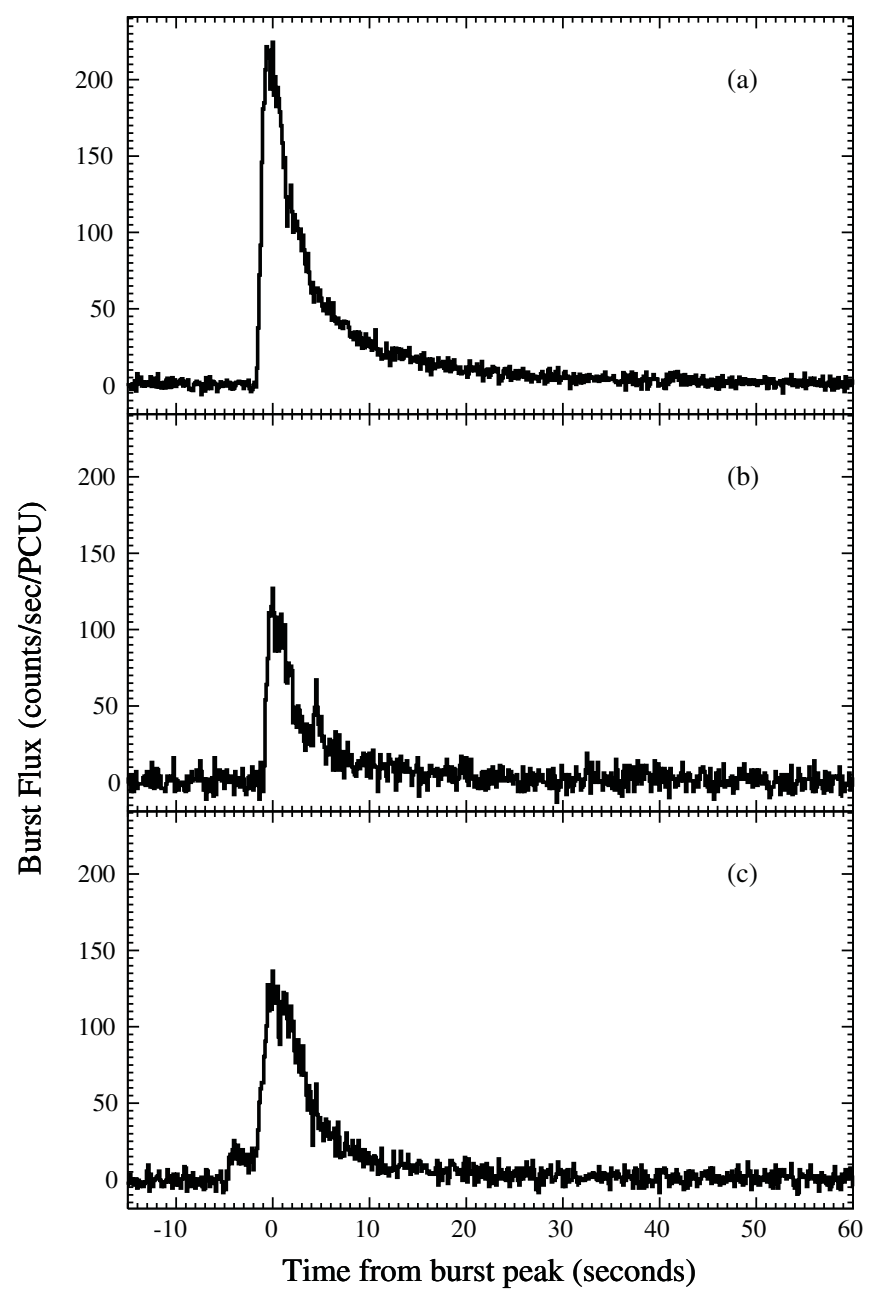

Figure 6. Thermonuclear X-ray bursts observed from 1A 1744-361 with RXTE. The light curves are background subtracted $0.125 \mathrm{~s}$ light curves generated using the full PCA energy band. The bursts were observed on 2005 July 16 (a), 2008 June 24 (b), and 2008 July 25 (c).

is used to characterize the central emission and a power law to model the Comptonized component. Such a two-component model provided an adequate fit for 1A 1744-361, however, we note that the properties of the Fe XXVI were insensitive to our specific choice of continuum model. Using our continuum model, we measured a total X-ray luminosity of $L_{X}=(1.94 \pm$ $0.02) \times 10^{36} \mathrm{erg} \mathrm{s}^{-1}$ in the $1-30 \mathrm{keV}$ band, assuming a distance of $d=9 \mathrm{kpc}$ as determined by Bhattacharyya et al. (2006a).
Church \& Bałucińska-Church (2004), using data from several dipping LMXBs, determined that the total 1-30 keV X-ray luminosity is directly related to the size of the ADC:

$$
r_{\mathrm{ADC}}=10^{8}\left(\frac{L_{X}}{10^{35} \mathrm{erg} \mathrm{s}^{-1}}\right)^{0.88 \pm 0.16} \mathrm{~cm} .
$$

Thus, using Equation (6), we find that the size of 1A 1744-361's $\mathrm{ADC}$ is $r_{\mathrm{ADC}}=(1.1 \pm 0.5) \times 10^{9} \mathrm{~cm}$. According to Church \& Bałucińska-Church (2004), the radius of the accretion disk, $r_{\text {disk }}$, is related to the radius of the ADC by

$$
r_{\mathrm{disk}}=r_{\mathrm{ADC}}\left(\frac{2 P}{2 \pi \Delta t}\right),
$$

where $P$ is the orbital period of the source and $\Delta t$ is the dip ingress time. Using two closely spaced dip episodes, Bhattacharyya et al. (2006a) were able to estimate the orbital period of 1 A $1744-361$ as $97 \pm 22$ minutes. $^{7}$ We found no additional dip episodes in the $R X T E$ data, and only a single short ( $\sim 2 \mathrm{~s})$ dip in the Chandra data on 2008 July 5. All the dips had durations in the $2-25 \mathrm{~s}$ range. Taking $2 \mathrm{~s}$ as an upper limit on the ingress time, together with our lower bounds on both the orbital period and $r_{\mathrm{ADC}}$, we find, using Equation (7), that the accretion disk has a radius of $r_{\text {disk }}>4.3 \times 10^{11} \mathrm{~cm}$. Thus, for 1A $1744-361$ the ADC covers $<0.3 \%$ of the accretion disk.

\subsection{The Fe XXVI Absorption Feature and Constraints on the Geometry of the System}

Although each individual spectrum of 1A 1744-361 was well fitted by a simple continuum model, the residuals showed clear evidence for an absorption feature at $6.961 \mathrm{keV}$ in the first and third Chandra observations. This feature is well resolved in the combined spectrum. We place an upper limit on the velocity of a redshifted flow of $v<221 \mathrm{~km} \mathrm{~s}^{-1}$. We measure a line width of $\Delta E=15.5 \pm 2.3 \mathrm{eV}$. Under the assumption that the line is broadened due to thermal effects, the measured line width corresponds to a dynamic temperature $k T=254 \pm 2 \mathrm{keV}$. Using this temperature, we performed a COG analysis for Fe XXVI and determined a column density for Fe XXVI of $N_{\text {Fe xxvI }}=7.4_{-1.0}^{+1.1} \times 10^{17} \mathrm{~cm}^{-2}$. We do not find statistical evidence for a feature at the Fe XXV energy. However, our upper limit on the equivalent width of an underlying Fe XXv

\footnotetext{
7 Note, however, that these dip episodes were separated by a data gap, thus not precluding a shorter period.
} 
feature allows us to place a lower limit on the ionization parameter of $\xi>10^{3.6} \mathrm{erg} \mathrm{cm} \mathrm{s}^{-1}$, as determined by our XSTAR simulations. Furthermore, our limit on $\xi$ allows us to constrain the geometry of the system. According to Tarter et al. (1969),

$$
\xi=\frac{L}{n_{e} r^{2}},
$$

where $L$ is the luminosity of the source (see Table 1), $r$ is the distance from the central object to the absorber, and $n_{e}$ is the electron number density given by

$$
n_{e}=\frac{N_{e}}{\Delta r} .
$$

Here, $N_{e}$ is the electron column density and $\Delta r$ is the thickness of the absorbing slab. From Equation (8), we find a distance to the absorber of $r \sim 2 \times 10^{11} \mathrm{~cm}$, if we assume a scale height of $\Delta r / r \sim 1$. This distance is safely within the accretion disk radius found earlier. Given that this distance is only an upper limit, an important question is whether it is possible that the absorption line is emanating from within the ADC. Taking $r=r_{\mathrm{ADC}}$, we find $\Delta r=0.006 r=6.4 \times 10^{6} \mathrm{~cm}$. For such a short scale height, and given the plausible inclinations for the system, it is hard to reconcile observing both the feature and dipping. Thus, it would appear that the feature is emanating outside of the ADC. Velocity broadening also places the absorber at $>2 r_{\text {ADC }}$.

\subsubsection{Cylindrical Geometry?}

Narrow absorption features have been seen almost exclusively in dipping LMXBs. This, together with the lack of evidence of orbital variability in the features, except during dipping, has led authors such as Boirin et al. (2004) to suggest that the absorbing material is distributed in a cylindrical geometry. In such a configuration, all LMXBs would exhibit absorption features, but would be observed preferentially in dippers because of their nearly edge-on geometry (Díaz Trigo et al. 2006; Boirin et al. 2005). Here, we discuss what such a geometry would imply for 1A 1744-361. If the incident angle is just grazing the top of this cylindrical distribution of absorbing material, then that could explain why the dips are so sparse and why even a small excess in continuum emission can render the absorption feature so difficult to detect. In this scenario, the height of this cylindrical distribution is given by $\Delta r=r \sin \theta$, where $60^{\circ}<\theta<80^{\circ}$. This, together with Equations (8) and (9), yields $r \lesssim 2 \times 10^{11} \mathrm{~cm}$. Note that given that we only have a lower limit on $\xi$, the absorber can actually be much closer. The fact that this value for $r$ is safely within the accretion disk radius (see Equation (7)), together with the fact that this scenario can explain, albeit in a qualitative way, the properties of the source, is very intriguing and worthy of further study with future observations.

\subsubsection{Variability in the Fe XXVI Feature?}

It is curious that the Fe XXVI feature is clearly present in all the Chandra observations except in the 2006 July 6 observation. This discrepancy cannot be due to dipping as only a single 2 s long dip was present in all the Chandra observations-too short to significantly affect the total spectrum. Furthermore, the Chandra observation containing the dip showed evidence for the Fe XXVI feature.

The properties of the continuum during the 2006 July 6 observation may provide insight into the cause of the variability of the FexXVI. The spectrum of the 2006 July 6 observation has a harder power-law index and a higher power-law flux than the other observations (see Table 1). A harder spectrum with a weaker Fe XXVI feature, indicative of a much more ionized absorber, is reminiscent of the behavior exhibited by the microquasar GRS $1915+105$. Neilsen \& Lee (2009) found that the equivalent width of the Fe XXVI feature in GRS $1915+105$ decreases as the hardness of the continuum increases. They suggested that the absorption features in GRS $1915 \pm 105$ emanate from an accretion disk wind and that in the harder states the corona photoionizes the wind, making it transparent, thereby reducing the equivalent width of the lines (see Neilsen \& Lee 2009). Although this interpretation is intriguing, line spectra due to accretion disk winds, although common from black hole systems, are virtually never seen in neutron star systems. However, a blueshifted line due to an accretion disk wind was observed by Miller et al. (2011) in the transient pulsar IGR J17480-2466. Also, a similar scenario has been suggested to explain the properties of the neutron star systems IGR J00291+5934 (Paizis et al. 2005) and 1A 0535+262 (Reynolds \& Miller 2010). Although in Sections 3.2 and 3.2.1 we show that even for different geometries the feature is most likely emanating far from the central source, unfortunately, we only have an upper limit on the flow velocity of the line. However, if we assume that the lines are emanating from a disk wind, we can use the line to place constraints on the properties of such a wind. The luminosity of the wind is given by $L_{\mathrm{W}}=(1 / 2) \dot{m}_{\mathrm{W}} v^{2}$, where $v$ is the flow velocity and $\dot{m}_{\mathrm{W}}$ is the mass flow rate of the wind given by $\dot{m}_{\mathrm{W}}=4 \pi r^{2} n_{e} m_{p} v(\Omega / 4 \pi)$, where $m_{p}$ is the proton mass and $\Omega / 4 \pi$ is the covering fraction. We can use Equation (8) to write the wind luminosity and wind mass flow rate as

$$
L_{\mathrm{W}}=2 \pi m_{p} v^{3}\left(\frac{\Omega}{4 \pi}\right)\left(\frac{L}{\xi}\right)
$$

and

$$
\dot{m}_{\mathrm{W}}=4 \pi m_{p} v\left(\frac{\Omega}{4 \pi}\right)\left(\frac{L}{\xi}\right),
$$

respectively. Using Equations (10) and (11), we find that 1A $1744-361$ has a ratio of wind to accretion luminosity of $L_{\mathrm{W}} / L_{\mathrm{acc}} \lesssim 2.8 \times 10^{-5}(\Omega / 4 \pi)$, and a ratio of wind mass flow rate to mass accretion rate of $\dot{m}_{\mathrm{W}} / \dot{m}_{\text {acc }} \lesssim 11(\Omega / 4 \pi)$. Thus, even for small covering fractions, the mass flow rate of the wind can be comparable to the accretion rate. Interestingly, these fractions are in fact higher than what Lee et al. (2002) found for GRS $1915+105,{ }^{8}$ specifically, $L_{\mathrm{W}} / L_{\text {acc }} \lesssim 7.4 \times 10^{-7}(\Omega / 4 \pi)$ and $\dot{m}_{\mathrm{W}} / \dot{m}_{\text {acc }} \lesssim 1.3(\Omega / 4 \pi)$. With our upper limit on the flow velocity $(v)$ and lower limit on the ionization parameter $(\xi)$, we cannot rule out the possibility that the line emanated from a wind. However, notice that the ratios $L_{\mathrm{W}} / L_{\text {acc }}$ and $\dot{m}_{\mathrm{W}} / \dot{m}_{\mathrm{acc}}$ are functions of $v$ and $\xi$. Therefore, it is not clear why source shows similar behavior to the microquasar GRS 1915+105, while other dipping LMXBs, having comparable values for $v$ and $\xi$, do not. Furthermore, IGR J17480-2446, which shows clear evidence of a wind, has $L_{\mathrm{W}} / L_{\mathrm{acc}} \approx 2.7 \times 10^{-2}(\Omega / 4 \pi)$ and $\dot{m}_{\mathrm{W}} / \dot{m}_{\mathrm{acc}} \approx 52(\Omega / 4 \pi)($ see Miller et al. 2011)—much higher than $1 \mathrm{~A} 1744-361$.

\footnotetext{
8 Note that the accretion luminosity for GRS $1915+105$, as a black hole system, is given by $L_{\mathrm{acc}}=\eta \dot{m}_{\mathrm{acc}} c^{2}$, where Lee et al. (2002) assumed an efficient of $\eta \sim 0.1$. For the neutron star systems

1A 1744-361 and 1A $0535+262$, we assumed a disk luminosity of $0.5 G M \dot{m}_{\mathrm{acc}} / R$ for the accretion luminosity.
} 


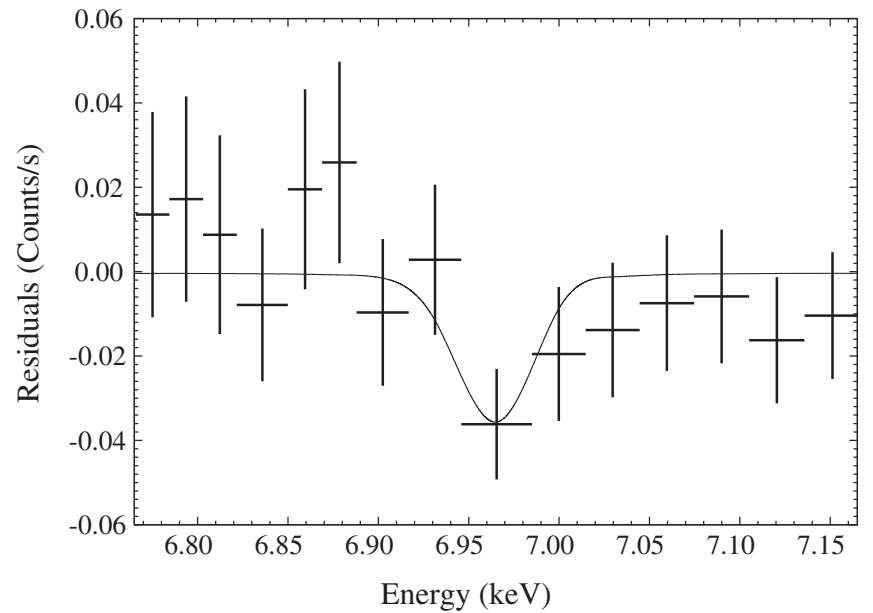

Figure 7. Error bars are the residuals after subtracting the best-fit continuum model from the spectrum of the 2006 July 6 Chandra observation. The smooth curve is the best-fit Gaussian emission line. The spectrum of the 2006 July 6 showed no significant deviations in its residuals after subtracting the best-fit continuum model. However, fitting this spectrum with a continuum and line models line properties that are consistent with those of the other observations. Thus, we cannot rule out the possibility that the line was present in this observation as well, but rendered indiscernible because of changes in its continuum emission.

As intriguing as a disk wind interpretation is, we caution that it is possible that this additional continuum emission at the higher end of the spectrum could have made any underlying Fe XXVI feature seem less significant. To verify this, we refit the 2006 July 6 spectrum, including an Fe XXVI absorption feature, and holding the line centroid and width fixed at the values found from the total spectrum. The normalization of the line and all the parameters of the continuum were free to vary during the fit. The best-fit model is shown in Figure 7. The value of the resulting normalization of the line is consistent, within the $1 \sigma$ uncertainties, with that of the total spectrum. Furthermore, despite the change in the continuum, the equivalent width of the feature is not significantly different (within the $1 \sigma$ uncertainties). Therefore, we conclude that we do not have the statistics to concretely judge whether the properties of the line truly changed during the 2006 July 6 observation, or whether the line remained the same but was indiscernible in this observation because of the excess continuum emission.

\subsection{Variability and Dipping}

Competing models have been put forth to explain the variations in the properties of the continuum and absorption lines during dipping and non-dipping intervals. It has been proposed that these variations are due to progressive and partial covering of an extended ADC by an extended absorber (see BałucińskaChurch et al. 2011; Church et al. 2005, 1998, for example). Authors such as Boirin et al. (2005) and Díaz Trigo et al. (2006), on the other hand, propose that an ionized absorber can explain dipping and non-dipping intervals, without the need of an extended ADC. Unfortunately, with only a single short ( $2 \mathrm{~s})$ dip in our Chandra observations, we cannot compare the spectral properties of the continuum and the line during dipping and nondipping intervals. However, we did explore whether a partially covered power-law model would fit the continuum as well as a blackbody+power law. Such a model did indeed characterize the data just as well as a blackbody + power law. We note that the properties of the line, however, were insensitive to which continuum model we chose. Similar to the blackbody+power-law model, for the partially covered power law the only parameters that significantly changed during the 2006 July 6 observation were the index and normalization of the power-law component. Note that this observation showed no dips; therefore, if the properties of the feature indeed did vary during these observations, then that is definitely unusual, as Boirin et al. (2004) find that the absorption features show no variability with orbital phase-except during dipping intervals.

\subsection{A 1744-361 and Other Short Period Dippers}

The plausible orbital period of 97 minutes $(1.6 \mathrm{hr}$ ) for 1A 1744-361 makes it the second shortest orbital period for a dipping LMXB that shows narrow absorption lines. The shortest period X-ray dipper, XB 1916-053, has a period that is nearly half of that of 1A 1744-361. Compared to other short period dippers, such as XB 1916-053 $(P=0.83 \mathrm{hr})$ and $\mathrm{XB}$ $1323-619(P=2.94 \mathrm{hr})$, we find that $1 \mathrm{~A} 1744-361$ is a factor of 10 more luminous than these sources (see Balman 2009, Table 1). It may not be surprising then that we do not find statistical evidence for an Fe XXV feature in 1A 1744-361. Combining Equations (6)-(8), we find that for sources with similar periods, a more luminous source will have a much more ionized absorber. Indeed, the lower limit on $\xi$ for 1A 1744-361 is consistent with the upper limits found for these sources (see Juett \& Chakrabarty 2006; Balman 2009, for example).

\subsection{Implications for the $6.4 \mathrm{keV}$ Emission Feature Observed with RXTE}

In addition to the narrow absorption feature reported here, Bhattacharyya et al. (2006b) discovered a broad (0.6 keV) Fe emission line at $\sim 6 \mathrm{keV}$ in the PCA data of 1A 1744-361. The fact that we now also see a narrow absorption feature at $6.9 \mathrm{keV}$ in the source's spectrum could explain why the centroid of the broad emission feature was at $\sim 6 \mathrm{keV}$ rather than at the expected value of $6.4 \mathrm{keV}$. Bhattacharyya et al. (2006b) suggested that this broad emission line was actually a $6.4 \mathrm{keV}$ line, which had flux removed by a narrow absorption feature at $6.9 \mathrm{keV}$, and appeared at $6 \mathrm{keV}$ after being binned with $R X T E$ 's course spectral resolution. Unfortunately, no broad emission features were observed in the individual or combined Chandra observations, however, this could be due to the smaller area of Chandra as compared to RXTE. By combining additional observations, one may be able to observe both the Fe emission feature and the Fe XXVI feature in 1A 1744-361. Indeed, such a measurement was made for $4 \mathrm{U} 1323-62$ by Boirin et al. (2005), using XMM-Newton. If future high spectral resolution observations of 1A 1744-361 reveal such an emission feature, and it is relativistically broadened, then such measurements can potentially constrain the compactness of the star.

\section{CONCLUSIONS}

Using three pointed Chandra HETG observations of 1A 1744-361 during its latest outburst, we have discovered a significant $6.961 \mathrm{keV}$ absorption feature in its persistent spectrum. This is the first time such a narrow absorption feature has been observed in the spectrum of 1A 1744-361, and we identify this feature as an Fe XXVI line. The width of the line was found to be $\Delta E=15.5 \pm 2.4 \mathrm{eV}$, which corresponds to a dynamic temperature of $k T=254 \pm 2 \mathrm{keV}$. We place an upper limit on the velocity of a redshifted flow of $v<221 \mathrm{~km} \mathrm{~s}^{-1}$. The line had an equivalent width of $W_{E}=27_{-3}^{+2} \mathrm{eV}$, for which we find a column density of $N_{\mathrm{Fe}_{\mathrm{XXVI}}}=7 \pm 1 \times 10^{17} \mathrm{~cm}^{-2}$ via a COG 
analysis. We place a lower limit on the ionization parameter of $\xi>10^{3.6} \mathrm{erg} \mathrm{cm} \mathrm{s}^{-1}$. Using the source's luminosity and the properties of the dips, we determined that 1A 1744-361 has an $\mathrm{ADC}$ radius of $r_{\mathrm{ADC}}=(1.1 \pm 0.5) \times 10^{9} \mathrm{~cm}$ and an accretion disk radius of $r_{\text {disk }}>4.3 \times 10^{11} \mathrm{~cm}$. The ionization parameter returned consistent results for the location of the absorber, placing it within the accretion disk (close to the edge) but most likely outside of the ADC. We also argue that a cylindrical distribution for the geometry of the absorbing material is consistent with our results. Using additional RXTE data, we generated an updated color-color diagram for the source, which confirms the suggestion of Bhattacharyya et al. (2006b) that this source is an "atoll" source. We report two bursts in addition to the one reported by Bhattacharyya et al. (2006a). We find no evidence for a broad emission line in the Chandra data like the one discovered by Bhattacharyya et al. (2006b) using PCA data. Observations with X-ray-imaging telescopes such as Chandra during the source's next outburst may reveal further narrow absorption features, as well as broad emission features. Such observations may constrain the structure and geometry of the system and are definitely warranted.

We thank C. B. Markwardt for useful discussions and for providing support for his numerical integration algorithm. This work has been supported by NASA via an ADP grant and a Chandra Guest Observer grant, as well as by the National Science Foundation (US NSF grant AST 0708424). This research has made use of data obtained through the High Energy Astrophysics Science Archive Research Center Online Service, provided by the NASA/Goddard Space Flight Center.

\section{REFERENCES}

Balman, Ş. 2009, AJ, 138, 50

Bałucińska-Church, M., Dotani, T., Hirotsu, T., \& Church, M. J. 2009, A\&A, 500,873
Bałucińska-Church, M., Schulz, N. S., Wilms, J., et al. 2011, A\&A, 530, A102 Bhattacharyya, S., Strohmayer, T. E., Markwardt, C. B., \& Swank, J. H. 2006a, ApJ, 639, L31

Bhattacharyya, S., Strohmayer, T. E., Swank, J. H., \& Markwardt, C. B. 2006b, ApJ, 652, 603

Boirin, L., Méndez, M., Díaz Trigo, M., Parmar, A. N., \& Kaastra, J. S. 2005, A\&A, 436, 195

Boirin, L., Parmar, A. N., Barret, D., Paltani, S., \& Grindlay, J. E. 2004, A\&A, 418, 1061

Canizares, C. R., Davis, J. E., Dewey, D., et al. 2005, PASP, 117, 1144

Carpenter, G. F., Eyles, C. J., Skinner, G. K., Wilson, A. M., \& Willmore, A. P. 1977, MNRAS, 179, 27P

Church, M. J., \& Bałucińska-Church, M. 2004, MNRAS, 348, 955

Church, M. J., Balucinska-Church, M., Dotani, T., \& Asai, K. 1998, ApJ, 504, 516

Church, M. J., Reed, D., Dotani, T., Bałucińska-Church, M., \& Smale, A. P. 2005, MNRAS, 359, 1336

Davison, P., Burnell, J., Ives, J., Wilson, A., \& Carpenter, G. 1976, IAU Circ., 2925, 1

Díaz Trigo, M., Parmar, A. N., Boirin, L., Méndez, M., \& Kaastra, J. S. 2006, A\&A, 445, 179

Emelyanov, A. N., Aref'ev, V. A., Churazov, E. M., Gilfanov, M. R., \& Sunyaev, R. A. 2001, Astron. Lett., 27, 781

Frank, J., King, A. R., \& Lasota, J.-P. 1987, A\&A, 178, 137

Iaria, R., Di Salvo, T., Lavagetto, G., Robba, N. R., \& Burderi, L. 2006, ApJ, 647,1341

Iaria, R., Lavagetto, G., D’Aí, A., di Salvo, T., \& Robba, N. R. 2007, A\&A, 463,289

Jiminez-Garate, M. A., Schulz, N. S., \& Marshall, H. L. 2003, ApJ, 590, 432

Juett, A. M., \& Chakrabarty, D. 2006, ApJ, 646, 493

Kallman, T., \& Bautista, M. 2001, ApJS, 133, 221

Kotani, T., Ebisawa, K., Dotani, T., et al. 2000, ApJ, 539, 413

Lee, J. C., Reynolds, C. S., Remillard, R., et al. 2002, ApJ, 567, 1102

Miller, J. M., Maitra, D., Cackett, E. M., Bhattacharyya, S., \& Strohmayer, T. E. 2011, ApJ, 731, L7

Neilsen, J., \& Lee, J. C. 2009, Nature, 458, 481

Paizis, A., Nowak, M. A., Wilms, J., et al. 2005, A\&A, 444, 357

Reynolds, M. T., \& Miller, J. M. 2010, ApJ, 723, 1799

Rybicki, G. B., \& Lightman, A. P. 1979, Radiative Processes in Astrophysics (New York: Wiley)

Tarter, C. B., Tucker, W. H., \& Salpeter, E. E. 1968, ApJ, 156, 943

Xiang, J., Lee, J. C., Nowak, M. A., Wilms, J., \& Schulz, N. S. 2009, ApJ, 701, 984 